\title{
Adapting Integrity Checking Techniques for Concurrent Operation Executions
}

\author{
Xavier Oriol and Ernest Teniente \\ Department of Service and Information System Engineering \\ Universitat Politècnica de Catalunya - BarcelonaTech \\ \{xoriol, teniente\}@essi.upc.edu
}

\begin{abstract}
One challenge for achieving executable models is preserving the integrity of the data. That is, given a structural model describing the constraints that the data should satisfy, and a behavioral model describing the operations that might change the data, the integrity checking problem consists in ensuring that, after executing the modeled operations, none of the specified constraints is violated.

A multitude of techniques have been presented so far to solve the integrity checking problem. However, to the best of our knowledge, all of them assume that operations are not executed concurrently. As we are going to see, concurrent operation executions might lead to violations not detected by these techniques.

In this paper, we present a technique for detecting and serializing those operations that can cause a constraint violation when executed concurrently, so that, previous incremental techniques, exploiting our approach, can be safely applied in systems with concurrent operation executions guaranteeing the integrity of the data.
\end{abstract}

Keywords: Integrity Checking, Concurrent Operations, UML/OCL

\section{Introduction}

One of the main challenges for achieving executable models is ensuring data integrity [1. That is, given a structural model describing the data and its integrity constraints, such as an UML diagram with OCL invariants; and a behavioral model describing the operations that can change this data, like OCL operation contracts for instance, the integrity checking problem consists in assessing whether the particular execution of a given operation in the current data state may induce a constraint violation. The difficulty of this problem is clear since, in the context of SQL databases, the integrity checking problem was already defined more than 25 years ago (under the form of SQL assertions checking [2]) and, still, none of the current major database management systems has implemented a solution for it (Oracle, SQL Server, DB2, PostgreSQL, MySQL).

As an example, consider the structural model described in Figure 1, written in UML/OCL, of a system for managing a research group. In this system, we have some researchers who work in some projects. Moreover, some of these researchers

Oriol, X.; Teniente, E. Adapting integrity checking techniques for concurrent operation executions. A: System Analysis and Modelling Conference. "System Analysis and Modeling: Languages, Methods, and Tools for Industry 4.0, 11th International Conference, SAM 2019: Munich, Germany, September 16-17, 2019: proceedings". Berlín: Springer, 2019, p. 235-248.

The final authenticated version is available online at https://doi.org/10.1007/978-3-030-30690-8_14 
lead some of these projects, although a project might have a maximum of two leaders. The OCL invariants states that researchers and projects are identified by their name (ResercherPK, and ProjectPK invariants), a leader of a project is also a member of the project (LeaderIsMember invariant), and that the salary of a leader of a project is higher than the salary of all its members (LeaderEarnsMore invariant). Note that these constraints might be violated because of the actions of the operations, as they are specified in the behavioral model.

\begin{tabular}{|c|c|c|c|c|}
\hline \multirow{3}{*}{\begin{tabular}{|l}
\multicolumn{1}{|c}{ Researcher } \\
$\begin{array}{l}\text { name: String } \\
\text { salary: Integer }\end{array}$
\end{tabular}} & \multirow{2}{*}{\begin{tabular}{|l}
$1 . *^{*}$ \\
member \\
$1 . .2$ \\
\end{tabular}} & \multirow[b]{2}{*}{ Leads } & \multirow[b]{2}{*}{$*$} & \multirow{3}{*}{$\frac{\text { Project }}{\text { name: String }}$} \\
\hline & & & & \\
\hline & leader & & & \\
\hline \multicolumn{5}{|c|}{$\begin{array}{l}\text { context Researcher inv ResearcherPK: } \\
\text { Researcher.allInstances()->isUnique(name) }\end{array}$} \\
\hline \multicolumn{5}{|c|}{$\begin{array}{l}\text { context Project inv ProjectPK: } \\
\text { Project.allInstances()->isUnique(name) }\end{array}$} \\
\hline \multicolumn{5}{|c|}{$\begin{array}{l}\text { context Project inv LeaderIsMember: } \\
\text { self.member->includesAll(self.leader) }\end{array}$} \\
\hline
\end{tabular}

Fig. 1. Structural model of a research group management system

In Figure 2, we show a fragment of the behavioral model for this system. In this model, we show the operation contracts, written in OCL, of four operations. The first one is required for adding new researchers (hireResearcher), the second one for assigning a leader to a project (addLeader), the third one for including a member in a project (addMember), and the last one for removing a member from a project (removeMember) ${ }^{1}$.

Depending on the current state of the information base, executing some of these operations with certain parameters can lead to a constraint violation. For instance, if we execute addLeader with parameters Mary and ModelsProject, but Mary is not currently a member of ModelsProject, the execution of the operation violates the LeaderIsMember constraint. The difficulty of this problem scales rapidly when complicating the operations and constraints involved.

To solve this problem, several proposals have been made in the modeling community based on incremental techniques 3/4/5/6 7. Briefly, incremental integrity checking techniques are based on the idea that, assuming that the current data state satisfies all the constraints, they check whether the data updated by

\footnotetext{
${ }^{1}$ This behavioral schema is oversimplified in purpose for the seek of facilitating the explanation of the method. More in general, our method can deal with operations applying several insertions/deletions at the same time, and not just one. In addition, our method is independant with the preconditions defined, thus, more complicated contracts are allowed. The unique requirement, as it will be explained laterly, is that OCL postconditions should be rewrittable in first-order logics (i.e., no aggregation nor transitive closure are allowed in postconditions).
} 


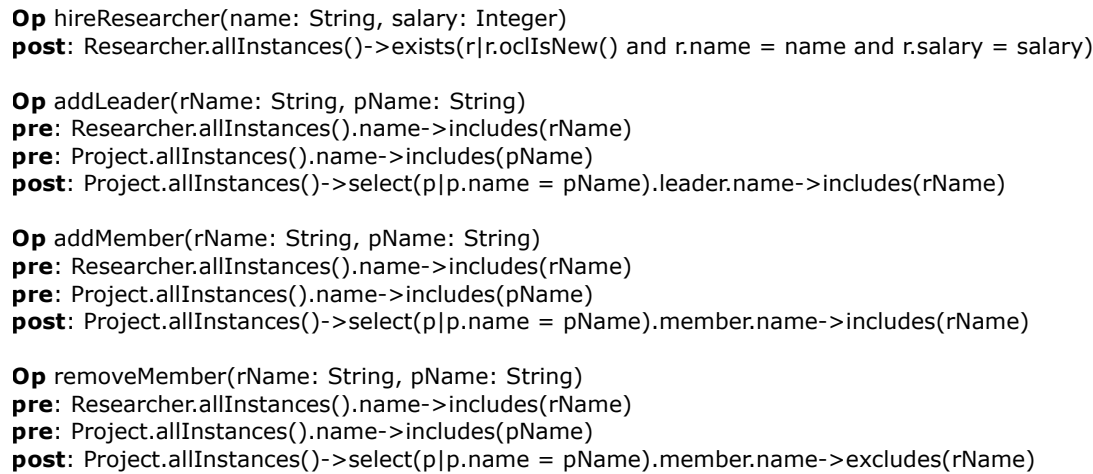

Fig. 2. Behavioral model of a research group management system

an operation execution leads to a violation without inspecting the rest of the data. For instance, following our previous example, we would only need to check whether Mary is a member of ModelsProject and, thus, there is no need to check other project leaders such as John, since John is not affected by the update.

However, to the best of our knowledge, all the presented techniques assume that operations are executed isolatedly, and thus, are not able to detect integrity violations when two operations executed concurrently interacts in a way that cause a constraint violation. For instance, assume that in our current data state Mary is a member of ModelsProject. In this situation, executing the operation to make Mary a leader of the project does not violate a constraint. In the same situation, executing, instead, an operation to remove Mary from the ModelsProject does not violate a constraint either. However, when executing both operations simultaneously, both interacts in a way to reach a new state in which Mary leads a project where she is not a member of. Thus, they raise a constraint violation.

This means that, right now, if we use the previous incremental techniques with systems that admit concurrent operation executions, some violations are going to be missed (i.e., previous incremental checking techniques are not complete when considering concurrency). Clearly, the problem can be solved by forcing all the operations to be executed in a serialized manner, but this might heavily penalize the runtime efficiency of the system.

Fortunately, not all operations must be executed in a serialized manner to avoid these violations. Indeed, not all operations can interact to cause a constraint violation. For instance, operations addLeader and removeMember can interact to violate LeaderIsMember and must be serialized, but operations addLeader and hireResearcher cannot interact to violate any constraint, and thus, can be executed concurrently.

In this paper, we define a method for identifying, and serializing, those operation executions that can interact to cause a constraint violation, permitting the rest of operations to be executed concurrently. In this way, we allow using the 
previous incremental techniques in systems with concurrent operations, without the penalization of serializing every execution, neither loosing completeness. Our technique has been implemented in a tool [8] for executing UML/OCL models, thus, showing that it is feasible in practice. In any case, since the core of our technique is fully based on logics, it can be adapted and implemented in other model executor tools using UML/OCL $9 \mid 10$ or other modeling languages, provided that they can be translated into logics. In particular, our technique can be adapted to first-order expressive languages (aka relational algebra equivalent) such as SQL, and SPARQL.

It is worth to mention that our work is, somehow, similar to the one in [11]. In particular, [11] detects operations invoked in a wrong order due to CRUD inconsistencies (e.g. reading some information deleted). We argue that our method and theirs can be combined, since both deal with different problems due to concurrency. Note, additionally, that our work is about checking a constraint on runtime assuming concurrency, and not on verifying/validating the models at compile time such as [12].

\section{Basic Concepts and Notation}

We review some key concepts and the basics of the notation used in the paper.

Terms, atoms and literals A term $t$ is either a variable or a constant. An atom is formed by a $n$-ary predicate $p$ together with $n$ terms, i,e. $p\left(t_{1}, \ldots, t_{n}\right)$. We may write $p(\bar{t})$ for short. If all the terms $\bar{t}$ of an atom are constants, we call the atom to be ground. A literal $l$ is either an atom $p(\bar{t})$, a negated atom $\neg p(\bar{t})$, or a built-in literal $t_{i} \omega t_{j}$, where $\omega$ is an arithmetic comparison (i,e. $<, \leq,=, \geq,>, \neq$ ).

Derived/Base predicates A predicate $p$ is said to be derived if the boolean evaluation of an atom $p(\bar{t})$ depends on some derivation rules, otherwise, it is said to be base. A derivation rule has the form: $\forall \bar{t} . p\left(\overline{t_{p}}\right) \leftarrow \phi(\bar{t})$ where $\overline{t_{p}} \subseteq \bar{t}$. In the formula, $p\left(\overline{t_{p}}\right)$ is an atom called the head of the rule and $\phi(\bar{t})$ is a conjunction of literals called the body. We suppose all derivation rules to be safe (i.e. all the variables appearing in the head or in a negated or built-in literal of the body also appears in a positive literal of the body) and non-recursive. Given several derivation rules with predicate $p$ in its head, $p(\bar{t})$ is evaluated to true if and only if one of the bodies of such derivation rules is evaluated to true.

Logic Formalization of the UML Schema. As proposed in [13] we formalize each class $C$ in the class diagram with attributes $\left\{A_{1}, \ldots, A_{n}\right\}$ by means of a base atom $c\left(\mathrm{Oid}, A_{1}, \ldots, A_{n}\right)$, each association $R$ between classes $\left\{C_{1}, \ldots, C_{k}\right\}$ by means of a base atom $r\left(C_{1}, \ldots, C_{k}\right)$ and, similarly, each association class $R$ between classes $\left\{C_{1}, \ldots, C_{k}\right\}$ and with attributes $\left\{A_{1}, \ldots, A_{n}\right\}$ by means of a base atom $r\left(\right.$ Oid, $\left.C_{1}, \ldots, C_{k}, A_{1}, \ldots, A_{n}\right)$.

Roughly speaking, when an object/relation encoded as $P(\bar{x})$ exists in some data state, the ground literal $P(\bar{x})$ evaluates to true in such data state. Conversely, when an object/relation encoded as $P(\bar{x})$ does not exists in some data state, the ground literal $P(\bar{x})$ evaluates to false in such data state. 


\section{Our Approach}

Our approach is based on the notion of structural events. A structural event is an elementary change in the population of the data, that is, an insertion or deletion of a class/association instance. For instance, inserting Leads(Mary, ModelsProject), or deleting WorksIn(Mary, ModelsProject) are structural events. For our purposes, we encode insertion structural events with the prefix ins, and deletion structural events with the prefix del, e.g., the previous structural events are encoded as ins_Leads(Mary, ModelsProject), and del_WorksIn(Mary, ModelsProject), respectively. Attribute updates can be seen as an insertion/deletion of the same object.

Executing an operation leads to structural events in the data, and these structural events might change the evaluation of a constraint, that is, the structural events might violate a constraint, or even repair a constraint that was going to be violated. For instance, executing the operation addLeader causes the structural event ins_Leads that might violate LeaderIsMember; on the contrary, executing the operation $a d d \_M e m b e r$ causes the structural event ins_WorksIn that might repair such violation.

The operations that must be serialized depend on the time where the chosen integrity checking technique takes place. In essence, the integrity checking techniques can be applied before executing the structural events (such as [3]), which we refer as precondition-time checking; or after it (such as [7), which we refer as postcondition-time checking. In the first case, we need to serialize two operations $O_{1}, O_{2}$ that can interact to cause a violation; on the second, we need to serialize two operations $O_{1}$ and $O_{2}$ if the structural events of $O_{1}$ might compensate the effects of $O_{2}$, since a rollback of $O_{1}$ might affect the consistency of $O_{2}$.

For instance, consider the operations addLeader, removeMember, and addMember from our running example. Using a preconditiom-time checking, the operations addLeader and removeMember should never be applied concurrently since they might interact to cause a constraint violation, and the checking technique will not realize of it since it makes the analysis separately. Note, however, that a postcondition-time checking will find the violation since, at the time of performing the analysis, both operations have been executed and all their effects are in the information system (and thus, at the time of checking the consistency of the data, the postcondition-time checking can find a leader not being a member of its project). However, in the case of a postconditiom-time checking, the operations that should not be executed (or at least analyzed) together are addMember and addLeader, since a rollback (or not) of the first might imply a violation (or not) of the second operation. Indeed, if we execute addMember and addLeader, and analyze together the consistency of the data, we might find that addLeader does not violate the LeaderIsMember constraint because the operation addMember adds the new leader as a member for the project, but if addMember violates any other constraint and must rollback, this rollback makes addLeader violate the LeaderIsMember. Thus, we should analyze the consistency of addLeader after the consistency analysis of addMember. Note that this problem does not occur in precondition-time checking techniques. 
Formally, when dealing with integrity checking in systems with concurrent operations, we identify two kinds of concurrency interactions between operations that must be taken into account:

- Potential concurrency violation. There is a potential concurrency violation between two operations $O_{1}$ and $O_{2}$ if, for some constraint $C$, the structural events applied by $O_{1}$ and $O_{2}$ might violate $C$.

- Potential concurrency compensation. There is a potential concurrency compensation from $O_{1}$ to $O_{2}$ if, for some constraint $C$, the structural events applied by $O_{1}$ might repair a violation of $C$ caused by the structural events of $\mathrm{O}_{2}$.

In the case of precondition-time checking, we must serialize two operations $O_{1}$ and $\mathrm{O}_{2}$ if they have a potential concurrency violation; in the case of postconditiontime checking, two operations $O_{1}$ and $O_{2}$ must be serialized if $O_{1}$ has a potential concurrency compensation with $\mathrm{O}_{2}$.

In this paper we focus on detecting this kind of interactions, and we suggest a serialization to deal with the problems they can carry out. Other approaches different than serialization, or a more refined versions of serialization, can be studied, but they are left for further work.

To detect this kind of interactions, we apply the following steps: 1) given all the operation contracts $\mathcal{O}$, we detect the kind of structural events applied by each operation $O \in \mathcal{O}, 2)$ given all constraints $\mathcal{C}$, we detect all the kind of structural events that can violate/repair each $C \in \mathcal{C}, 3)$ for each pair of operations $O_{1}, O_{2}$, and each constraint $C$, we use the structural events to analyze if there is any kind of interaction between them w.r.t. $C$. Note that all these analysis can be performed at compile time since they purely rely on the model specification of the operations and constraints.

\subsection{Detecting the kind of structural events applied by some operation}

Given an OCL operation contract, it is possible to identify, at compile time, which are the kind of structural events applied by the operation [1415. For our purposes, we rely on the approach of [14 to detect them. In essence, the idea behind this approach is to translate any operation contract to an equivalent logic formula that, intuitively, states that executing of an operation implies the application of certain structural events.

In particular, the previous operations from Figure 2 can be encoded by means of the following logic formulas:

ins_Researcher (R, Name, Salary) :- hireResearcher (Name, Salary)

ins_Leads (R, P) :- addLeader (RN, PN), Researcher (R, RN, S), Project (P, PN)

del_WorksIn (R, P) :- removeMember (RN, PN), Researcher (R, RN, $S)$, Project (P, PN)

ins_WorksIn(R, P) :- addMember (RN, $P N)$, Researcher (R, RN, S), Project (P, PN)

Intuitively, the first formula states that invoking the operation hireResearcher with parameters Name, Salary causes the structural event of ins_Researcher(R, 
Name, Salary) to happen, where $R$ is a new object identifier value. The second one states that, when invoking the operation addLeader with parameters $R N$ and $P N$, there is a structural event ins_Leads $(R, P)$ provided that $\mathrm{R}$ and $\mathrm{P}$ are the researcher and project identified by $R N$ and $P N$, respectively. Similarly, the third formula states that, when invoking the operation removeMember, there is a $\operatorname{del} \operatorname{WorksIn}(R, P)$ structural event.

The rationale behind such translations can be sketched as follows. OCL operations such as includes, and includesAll, when used in a postcondition, are used to specify insertions on associations [16. The source of such operations represent the association where the insertion takes place, and the body of such operations represents the value/s inserted. Thus, the logic translation consists in, roughly speaking, 1) identifying these OCL operations, 2) generate an insertion rule for each one of them, and 3) put, in the generated logic rule, the OCL logic translation of the objects where the insertion takes place. For this last step, we have to translate into logics the source of the OCL operation (i.e., the objects where the insertion takes place), and translate the body of the OCL operation (i.e., the objects inserted). That is, if the source/body of the OCL operation is an object/s $x$, we have to build an OCL logic translation that retrieves all those $x$. In general, if OCL obtains some objects $x$ by means of $n$ navigation steps, the logic translation consists in a conjunction of $n$ non-ground ordinary literals, each one representing one step of the navigation. A similar approach is taken with OCL oclIsNew() and excludes operations as detailed in [14].

Thus, and thanks to this translation which is already implemented [17, the structural events implied by each operation become explicit in the head of each rule. Hence, we can build a program that reads this translation, and realizes that executing hireResearcher implies the structural event ins_Reseracher, addLeader implies ins_Leads, and removeMember implies del_WorksIn.

Note that, in general, an operation will apply more than one kind of structural event when executed. For instance, we could specify an operation that creates a new researcher and adds his membership associations. In this case, and following 14, an operation is translated into several logic formulas, each one implying a different structural event. Thus, the structural events implied by such operation is the union of all the structural events appearing in all the formulas.

\subsection{Detecting the structural events that violate/repair a constraint}

Given a constraint $C$, it is possible to determine, at compile time, which are the kind of structural events that might violate a constraint, and also those that may repair it [187]. For our purposes, we use the approach defined in [18 since it is based on logics in a similar way as we did in previous section.

In essence, we first translate the UML and OCL constraints into logic denials, that is, logic formulas stating the condition that rise a constraint violation. Following, for instance, the automatic translation of UML/OCL constraints to denials defined in [13, our running example would bring the following logic formulas: 


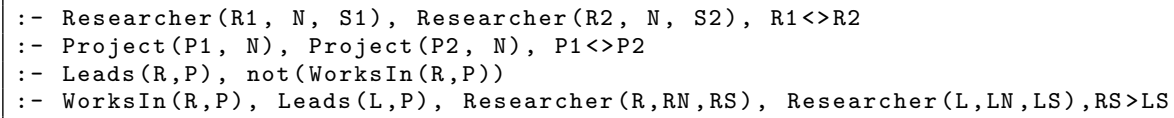

The first and second formulas, encode that, if there are two different researchers or projects with the same name, there is a constraint violation. The third one states a constraint violation if $R$ leads a project $P$ where s/he does not work in. The last formula asserts a violation if for some project $P$, there is a leader $L$ that earns less than a worker $R$.

The rationale behind such translations can be summarized as follows. Like in the previous section, every OCL value/set expression that retrieves an object/s $x$ is translated into a conjunction of ordinary literals. This conjunction of ordinary literals contains a variable $x$ that, roughly speaking, represents any object $x$ that can be retrieved from the navigation. Then, every OCL boolean operator that combines two OCL value/set expressions to retrieve a boolean value is translated as two conjunctions of ordinary literals (one for each OCL value/set expression) together the translation of the OCL operator. In the easiest case, like an OCL equality operator, the translation consists in a logic built-in literal. Other cases require more complex treatment, like the definition of derivation rules, as detailed in [13].

Given the logic formulas, we can realize which structural events might make these formulas true (and thus, rise a violation), and which of them might make them false (and thus, repair the violation).

To do so, we rely on the event rule equivalences [19. The event rule equivalences define when a structural event makes a literal true/false in the new state of the data after applying the events. In particular, consider $P^{N}$ to be the literal $P$ evaluated in the new data state. Then, the event rule equivalences tells us that:

$$
\begin{aligned}
P^{N}(\bar{x}) & \equiv i n s \_P(\bar{x}) \vee\left(P(\bar{x}) \wedge \neg d e l \_P(\bar{x})\right) \\
\neg P^{N}(\bar{x}) & \equiv d e l \_P(\bar{x}) \vee\left(\neg P(\bar{x}) \wedge \neg i n s \_P(\bar{x})\right)
\end{aligned}
$$

Intuitively, the literal $P(\bar{x})$ is true in the new state after applying the structural events if we have inserted $P(\bar{x})$ through some insertion structural event, or $P(\bar{x})$ was already true in the data state and we have not deleted it. Similarly, $\neg P(\bar{x})$ is true in the new state after applyng the structural events if we have deleted $P(\bar{x})$ through some deletion structural event, or $P(\bar{x})$ was already false in the data state and we have not inserted it.

Applying the previous equivalences to our logic denials, by means of applying all the possible literal substitutions (aka unfoldings) given by the event rule equivalences, we obtain what we call event-dependency constraints (EDCs), that is, denials that tells which structural events rise a constraint violation. For instance, for the first denial we obtain: 


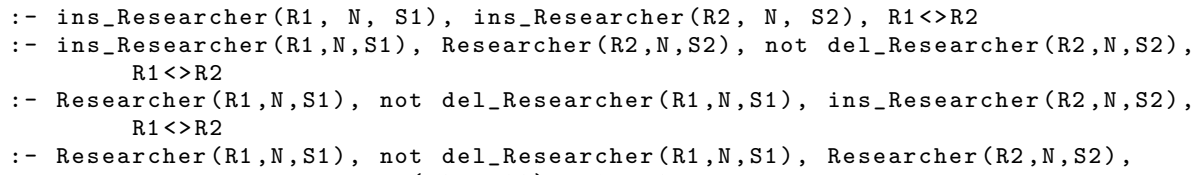

The first EDCs states that there is a constraint violation if we apply two different structural events for inserting a researcher with the same name. The second and third one specify that if we insert a new researcher with a name $N$, and this name $N$ belongs to some researcher in the current data, but we do not remove this researcher, there is a constraint violation. Finally, the last rule tells us that if we have two researchers with the same name and we do not remove any of them, there is a constraint violation.

Intuitively, the structural events that appear positively in an EDC are the structural events that might cause a violation, while those that appear negatively in an EDC are the structural events that might repair the violation (since they make the body of the EDC, which detects the violation, to evaluate to false). For instance, ins_Researcher is a structural event that can cause a violation of the ResearcherPK constraint, while del_Researcher is a structural event that can repair it.

It is worth to hightlight that the number of EDCs obtained from one denial grows exponentially with the lenght of the denial encoding. However, some optimizations can be applied to reduce the number and size of the denials 3 . Indeed, considering the classical optimization that the initial data state does not violate any constraint, and that there is homomorphism between denials two and three, the unique EDCs required are:

:- ins_Researcher (R1, N, S1), ins_Researcher (R2, N, S2), R1 <>R2

$:-$ ins_Researcher $(R 1, N, S 1)$, Researcher $(R 2, N, S 2)$, not del_Researcher $(R 2, N, S 2)$, $\mathrm{R} 1<>\mathrm{R} 2$

\subsection{Detecting operations and constraints interactions through the structural events}

At this point, we want to analyze, using the structural events previously determined, which kind of interactions might have two operations w.r.t. some constraint. To do so, and benefiting from the fact that all our approach is based on logics, we are going to use an unfolding technique. In essence, our idea is to unfold the body of the EDCs obtained in Section 3.2, which tells us which structural events cause a violation/repair, with the rules from Section 3.1, which specifies which structural events are implied by the operations. As a result, we obtain some new rules that directly define which operations can violate/repair some constraint.

For instance, if we unfold the previous EDCs with the logic rules that tells that hiring a researcher makes an insertion structural event of a researcher, we obtain: 
:- hireResearcher (N, S1), hireResearcher (N, S2)

$:-$ hireResearcher (N, S1), Researcher (R2, N,S2), not (del_Researcher $(R 2, N, S 2))$, $\mathrm{R} 1<>\mathrm{R} 2$

Intuitively, the first rule states that two executions of hireResearcher can interact to rise a constraint violation (i.e., a violation of ResearcherPk constraint). The second rule tells us that, hireResearcher might be compensated with an operation that deletes researchers. However, since there is no operation to delete researchers, there is no interaction according to this rule.

We now bring an example of a detection of a compensation interaction. Consider the EDCs obtained from the LeaderIsMember constraint:

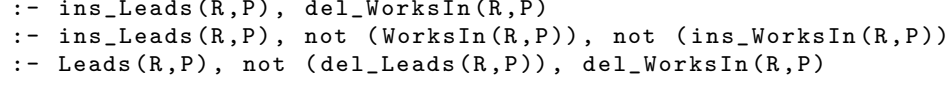

Intuitively, the first EDC states that there is a violation if we insert that $R$ is going to lead a project $P \mathrm{~s} /$ he is leaving. The second asserts a violation if we insert that $R$ is going to lead a project $P \mathrm{~s} /$ he is not working in and that he is not going to work in. Finally, the third EDC detects a violation if we delete $R$ from working in $P$, when $R$ is leading $P$ and we do not delete $R$ as a leader of $P$.

Then, when unfolding the EDCs according to the rules from Section 3.1 which encodes the operations behavior, we have:

$:-\operatorname{addLeader}(R N, P N)$, Researcher $(R, R N, S)$, Project (P, PN), removeMember (RN, PN) $:-\operatorname{addLeader}(R N, P N)$, Researcher $(R, R N, S)$, Project (P, PN), not (WorksIn $(R, P))$, not (addMember $(R N, P N)$ )

Roughly speaking, these rules are saying which operations can interact to cause/compensate a violation. For instance, the first rule says that, when applying the addLeader operation, between a researcher with name $R N$ and project named $P N$, over a database that contains that researcher and project, while applying the operation or removeMember with the same parameters, then, there is a constraint violation. Differently, the second rule says that, when applying the addLeader operation with the same parameters, over a database where the researcher does not work for the project, and without applying the addMember operation, then, there is another constraint violation.

In general, two operations that appears positively in the same denial have a potential concurrent violation, whereas two operations, where one appears in a negated literal, have a potential concurrent compensation. Indeed, we can identify that the operations addLeader and removeMember has a potential concurrent violation interaction with LeaderIsMember, since they both appear positively in the body of the first denial, while addLeader and addMember has a potential compensation interaction, since addMember appears negatively and addLeader positively in the same denial. Hence, addLeader and removeMember should be serialized for precondition-time checking techniques, whereas addMember and addLeader should be serialized (preferribly in this order) for postcontition-time techniques. 
Finally, we can summarize all the above with the following statement: $a$ precondition-time checking technique should serialize those operations appearing positively in the unfolded EDCs, whereas a postcondition-time checking technique should serialize those operations appearing, one as a positive literal and the other as a negative literal, in the unfolded EDCs.

\section{Implementation}

We have implemented our approach in OpExec [8], an artifact-centrict business process model executor. Briefly, this tool is capable of loading the structural and behavioral models of the system at compile time, encoded in logics, and, at runtime, execute the operations invoked by the user into a relational database.

In OpExec, we integrated an implementation of a precondition-time checking technique 20. This technique assumed that all operations were executed isolatedly, i.e., not concurrently, and thus, required an automatic serialization technique as the one we have discussed in this paper.

The implementation of our technique is summarized in Figure 3 . In OpExec, a user loads, in compile time, the structural and behavioral models into a Controller. Then, when the user wants to execute the models, the user uses the Controller to create a ProcessExecutor. The ProcessExecutor contains an artifactID, which is an id number to identify all the information related to such process. At runtime, the user invokes an operation from the behavioral model through the ProcessExecutor. This processExecutor, then, creates an OperationExecThread, which is a new Thread that will execute the operation invoked by the user into the database.

The integrity checking part is implemented in the OperationExec Thread which, intuitively, checks whether its structural events are going to violate any constraint according to the current contents of the data. In case that there is any constraint violation, the OperationExecThread does not commit any change into the database, otherwise, the database is updated accordingly.

In order to enable multiple users invoke OpExec concurrently, and to guarantee that the integrity checking part detects all possible violations, we implemented the OperationExecThreadManager. When a new OperationExecThread is created, this Thread is enqueued in the OperationExecThreadManager, which is responsible of executing it as soon as it is safe to execute it, i.e., when it is guaranteed that it will not interact, with any other currently running OperationExecThread, to cause a violation.

The technique discussed in our paper is fully implemented in the OperationExecThreadManager class. That is, at compile time, it receives the models and performs our interaction analysis to detect which operations can collaborate to raise a constraint violation. Then, at runtime, if we try to execute an operation which might interact with another operation which is currently being executed, the OperationExecThreadManager delays the execution of the first untill the second has finished. 


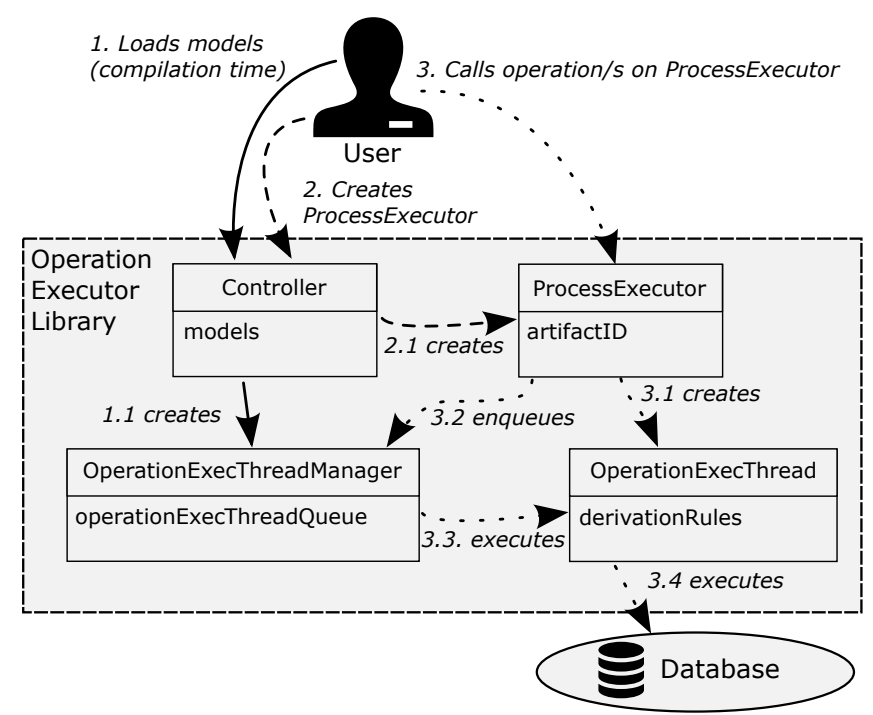

Fig. 3. Architecture of a Model Executor with an Integrity Checking Technique

Although our implementation is thought for a precondition-time integrity checking, we understand that it might not be difficult to adapt it to work with a postcondition-time integrity checking such as those presented in 44/51677.

\section{Conclusions}

We have presented an approach for adapting integrity checking techniques to systems with concurrent operations. Indeed, current integrity checking techniques do not take into account concurrent operation executions and, as we have seen, this concurrency might cause violations which cannot be detetected by these techniques.

To solve this situation, we have defined an approach for identifying which operations can bring problems to the integrity checking techniques when executed concurrently. As we have seen, the kind of operations that might bring problems depend on the kind of integrity checking technique applied. On the one hand, integrity checking techniques performed at precondition time should avoid concurrent executions of operations that might collaborate to cause a violation. On the other, integrity checking techniques performed at postcondition time should avoid analysing concurrently two operations if one compensates a violation from the other. Our approach can detect both kinds of interactions and thus, can be applied for both kinds of integrity checking techniques. To show the feasibility of our approach, we have implemented it in the OpExec model executor.

As further work, we would like to highlight the necessity of defining a UML/OCL benchmark for experimenting with concurrent operations, and thus, enable comparative efficiency experiments with other methods. 


\section{References}

1. Olivé, A., Cabot, J.: A research agenda for conceptual schema-centric development. In: Conceptual Modelling in Information Systems Engineering. Springer (2007) 319-334

2. ANSI Standard: The SQL 92 Standard. (1992)

3. Oriol, X., Teniente, E.: Incremental checking of OCL constraints with aggregates through SQL. In: Conceptual Modeling - 34th International Conference, ER 2015, Stockholm, Sweden, October 19-22, 2015, Proceedings. (2015) 199-213

4. Bergmann, G.: Translating ocl to graph patterns. In: Model-Driven Engineering Languages and Systems - 17th International Conference, MODELS 2014, Valencia, Spain, September 28 - October 3, 2014. Proceedings. (2014) 670-686

5. Uhl, A., Goldschmidt, T., Holzleitner, M.: Using an OCL impact analysis algorithm for view-based textual modelling. ECEASST 44 (2011)

6. Groher, I., Reder, A., Egyed, A.: Incremental consistency checking of dynamic constraints. In: Fundamental Approaches to Software Engineering. Springer (2010) 203-217

7. Cabot, J., Teniente, E.: Incremental integrity checking of UML/OCL conceptual schemas. Journal of Systems and Software 82(9) (2009) 1459-1478

8. De Giacomo, G., Oriol, X., Estañol, M., Teniente, E.: Linking data and BPMN processes to achieve executable models. In: Advanced Information Systems Engineering - 29th International Conference, CAiSE 2017, Essen, Germany, June 12-16, 2017, Proceedings. (2017) 612-628

9. Object Management Group (OMG): Unified Modeling Language (UML) Superstructure Specification, version 2.4.1. (2011) http://www .omg.org/spec/UML/.

10. Object Management Group (OMG): Object Constraint Language (UML), version 2.4. (2014) http://www.omg.org/spec/OCL/

11. Combi, C., Oliboni, B., Weske, M., Zerbato, F.: Conceptual modeling of interdependencies between processes and data. In: Proceedings of the 33rd Annual ACM Symposium on Applied Computing. SAC '18, New York, NY, USA, ACM (2018) 110-119

12. Przigoda, N., Hilken, C., Wille, R., Peleska, J., Drechsler, R.: Checking concurrent behavior in UML/OCL models. In: 18th ACM/IEEE International Conference on Model Driven Engineering Languages and Systems, MoDELS 2015, Ottawa, ON, Canada, September 30 - October 2, 2015. (2015) 176-185

13. Queralt, A., Teniente, E.: Verification and validation of UML conceptual schemas with OCL constraints. ACM TOSEM 21(2) (2012) 13

14. Queralt, A., Teniente, E.: Reasoning on uml conceptual schemas with operations. In van Eck, P., Gordijn, J., Wieringa, R., eds.: Advanced Information Systems Engineering, Berlin, Heidelberg, Springer Berlin Heidelberg (2009) 47-62

15. Cabot, J.: From declarative to imperative uml/ocl operation specifications. In Parent, C., Schewe, K.D., Storey, V.C., Thalheim, B., eds.: Conceptual Modeling - ER 2007, Berlin, Heidelberg, Springer Berlin Heidelberg (2007) 198-213

16. Cabot, J.: From declarative to imperative uml/ocl operation specifications. In Parent, C., Schewe, K.D., Storey, V.C., Thalheim, B., eds.: Conceptual Modeling - ER 2007, Berlin, Heidelberg, Springer Berlin Heidelberg (2007) 198-213

17. Oriol, X.: Verificació i validació d'esquemes conceptuals uml/ocl amb operacions. (2012)

18. Oriol, X., Teniente, E., Tort, A.: Computing repairs for constraint violations in uml/ocl conceptual schemas. Data \& Knowledge Engineering 99 (2015) 39 - 58 
Selected Papers from the 33rd International Conference on Conceptual Modeling (ER 2014).

19. Olivé, A.: Integrity constraints checking in deductive databases. In: Proceedings of the 17th Int. Conference on Very Large Data Bases (VLDB). (1991) 513-523

20. Oriol, X., Teniente, E., Rull, G.: TINTIN: a tool for incremental integrity checking of assertions in SQL server. In: Proceedings of the 19th International Conference on Extending Database Technology, EDBT 2016, Bordeaux, France, March 15-16, 2016, Bordeaux, France, March 15-16, 2016. (2016) 632-635 